\title{
Impact of Breast Cancer Diagnosis and Treatment on Work-Related Life and Financial Factors
}

\author{
Christiane Richter-Ehrenstein $^{\mathrm{a}} \quad$ Julia Martinez-Pader ${ }^{\mathrm{b}}$

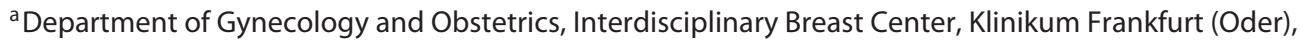 \\ Frankfurt (Oder), Germany; ${ }^{b}$ Department of Internal Medicine, DRK Krankenhaus Bremen, Bremen, Germany
}

\section{Keywords}

Breast cancer · Work · Employment · Survivorship · Adjuvant therapy · Finance

\begin{abstract}
Background: Survival rates of breast cancer patients are high, and the majority of women is $<65$ years old when first treated for breast cancer. The aim of our study is to assess work-related life factors and analyze the financial opportunities of breast cancer survivors and how this is reflected in the general quality of their lives. Methods: We conducted a cross-sectional, hospital-based monocentric questionnaire study of women diagnosed with breast cancer. Recruitment was carried out from January to March 2011 for women diagnosed with and treated for primary breast cancer between 2005 and 2010 at Charité University Hospital Berlin. Results: The study included 492 breast cancer patients without recurrence. In total, $81.3 \%$ of the women returned to work, and $30.2 \%$ of the women felt a reduction of financial opportunities. Financial problems were named by at least $20 \%$ of the patients as being the main cause for a reduced quality of life. Conclusion: Long-term, disease-free breast cancer survivors reported a significant change in their work-related factors as well as changes in their financial opportunities.
\end{abstract}

(C) 2020 S. Karger AG, Basel

\section{Introduction}

Breast cancer is the most common cancer among women worldwide [1-3]. Survival rates have improved significantly, especially in high-income countries, because of advancement in cancer diagnosis and treatment regimens [3-6]. With the increasing number of breast cancer survivors, the interest and need to support this group of patients has grown, and the awareness of the financial and economic impact of the disease is becoming a recognized field [79]. For survivors going back to work underlines the return to normal activities, social recovery, and a step towards an improved quality of life [10-12]. Most research to date has been from the USA where publicly funded healthcare is limited, and it is surprising how little is known about this topic in the majority of countries which operate with mixed public-private healthcare systems such as Denmark, the Netherlands, and numerous others.

So far the body of evidence is relatively small; for example, there is no data from Germany about the impact of breast cancer on survivors' work-related life and the surrounding financial factors. Hence, the main objective of our study is first to assess the work-related life of breast cancer survivors in terms of returning to work, the percentage of unemployment, and the rate of retirement, and second to analyze the financial opportunities of survivors and this reflection on their general quality of life. 
Table 1. Baseline demographic and clinical characteristics of the sample at the time of diagnosis

\begin{tabular}{lc}
\hline Education & \\
High school or less & $154 / 31.3 \%$ \\
Some college & $178 / 36.2 \%$ \\
College graduate or greater & $119 / 24.2 \%$ \\
Missing & $41 / 8.3 \%$ \\
\hline Marital status & \\
Married/partnership & $353 / 71.8 \%$ \\
Widowed & $59 / 12.0 \%$ \\
Not married & $80 / 16.2 \%$ \\
\hline Employment status & \\
No work & $41 / 8.3 \%$ \\
Retired & $178 / 36.1 \%$ \\
Working & $273 / 55.6 \%$ \\
\hline Type of work & \\
Worker & $31 / 11.4 \%$ \\
Employee & $157 / 57.5 \%$ \\
Officer & $31 / 11.4 \%$ \\
Self-employed & $26 / 9.5 \%$ \\
Graduate & $28 / 10.2 \%$ \\
\hline Surgery type & \\
Breast conservation & $330 / 67 \%$ \\
Mastectomy & $162 / 33 \%$ \\
\hline Radiation therapy & \\
Yes & $376 / 76.4 \%$ \\
No & $106 / 21.5 \%$ \\
Missing & $10 / 2.1 \%$ \\
\hline Chemotherapy & \\
Yes & $208 / 42.3 \%$ \\
No & $272 / 55.3 \%$ \\
\hline Antissing & $12 / 2.4 \%$ \\
Yes & \\
No & $210 / 42.9 \%$ \\
Missing & \\
\hline
\end{tabular}

\section{Methods}

\section{Patients and Procedure}

We conducted a cross-sectional, hospital-based monocentric study of women diagnosed with breast cancer at Charité University Hospital in Berlin, Germany. Enrolment in the study was carried out from January to March 2011 for women diagnosed with and treated for primary breast cancer between 2005 and 2010 at Charité University Hospital Berlin. Patients treated at the Charite Breast Center represent the common general breast cancer population in Germany. It consists of the local resident population and is heterogeneous in age, education, and financial resources. We mailed letters of invitation during the enrolment period and included the study questionnaire and a postage-paid return envelope with the mailing. Individuals were excluded if they could not complete a questionnaire in German. Because of the sensitive nature of the questions, questionnaires were completely anonymous, and therefore it was not possible to link the survey to the state and progress of the disease. In the given time period, Germany had not yet implemented a nationwide tumor registration, which could provide data on the disease progression of cancer patients centrally.
Questionnaire Design and Content

A questionnaire assessing the financial and work-related factors, which the breast cancer survivors had faced, was developed based on in-depth interviews with oncologists, social workers, and survivors of breast cancer in view of the existing literature. Information was sought on sociodemographic characteristics such as age, presence of dependent family members, education (high school or less, some college, college graduate or greater, missing), work-related factors such as employment status, time it took to return to work, changes in the workplace and working hours, type of work (no work, retired, housewife, worker, employee, officer, self-employed, graduate), changes in personal income, and financial situation. Clinical questions were also included to specify different interventions and therapies (breast-conserving therapy, mastectomy, radiation therapy, chemotherapy, and antihormonal therapy), as well as changes of the general quality of life. The draft questionnaire was pre-tested in 5 patients and showed the content validity of the survey.

The datasets used and/or analyzed during the current study are available from the corresponding author on reasonable request.

\section{Statistical Analysis}

All statistical analyses were conducted using the SPSS statistical software version 13 for MAC. Questionnaire results were collected and analyzed using descriptive statistics. All reported $p$ values were two-tailed, and the statistical significance was defined as $p<0.05$.

\section{Results}

A total number of 492 breast cancer patients participated in the survey. The mean age of all participants was 66 years (standard deviation \pm 11.8 ). Table 1 reports the baseline demographic and clinical characteristics of the sample. Regarding the clinical characteristics, $67 \%$ of the women received breast-conserving surgery, and $76.4 \%$ had radiation therapy. In total, $42.3 \%$ of the patients were treated with chemotherapy, and $54.7 \%$ of the women received antihormonal therapy. With respect to sociodemographic characteristics, $71.8 \%$ were married at the time of diagnosis, and more than $60 \%$ had graduated from high school or college. At the time of diagnosis, $55.6 \%$ of the patients were working, and $36.1 \%$ were retired. In the group of women who were working, $>70 \%$ were employed and 9.5\% were self-employed. Table 2 shows the work experience in detail. The majority of patients returned to work after 6 months, but $18.7 \%$ of the women never returned to work. In the group of patients returning to work before 6 months, 59.1\% were self-employed. With respect to the reduction of working hours, $>80 \%$ of the women who returned to work had no reduction of hours in their work timetable. With respect to the point of losing their job and being unemployed, 6.5\% of the women were unemployed after breast cancer diagnosis and treatment. Asked for their career opportunities in general after the disease, around $70 \%$ of the patients reported no change. Concerning the financial experience after the diagnosis of breast cancer, $82.4 \%$ of the patients 
Table 2. Employment experiences reported by breast cancer survivors

\begin{tabular}{lr}
\hline Return to work & \\
Before 8 weeks & $44 / 16.1 \%$ \\
Between 8 weeks and 6 months & $48 / 17.6 \%$ \\
After 6 months & $130 / 47.6 \%$ \\
Never returned to work & $51 / 18.7 \%$ \\
\hline Reduction of working hours & \\
Up to 2 h per week & $13 / 5.9 \%$ \\
2-4 h per week & $28 / 12.6 \%$ \\
No reduction & $181 / 81.5 \%$ \\
\hline Career opportunities in general & \\
Yes & $154 / 69.3 \%$ \\
No & $68 / 30.7 \%$ \\
\hline
\end{tabular}

Table 3. Financial experiences reported by breast cancer survivors

\begin{tabular}{lc}
\hline Personal income & \\
Decreased up to 5\% & $1 / 0.5 \%$ \\
Decreased up to $10 \%$ & $7 / 3.1 \%$ \\
Decreased up to $15 \%$ & $31 / 14 \%$ \\
No change & $183 / 82.4 \%$ \\
\hline
\end{tabular}

Financial situation

Reduction of financial opportunities

$67 / 30.2 \%$

No reduction

$155 / 69.8 \%$

reported no change in their personal income, but $30.2 \%$ felt a reduction of financial opportunities, as seen in Table 3 . This was more pronounced in the group of self-employed women in which $59.1 \%$ reported a decrease in their financial possibilities.

In the next step of the analysis, we looked for the association between different therapy modalities and work absence. In the group of patients who returned to work after 6 months, $80 \%$ of the patients received chemotherapy $(p<0.05), 57.9 \%$ had breast-conserving therapy, $62.6 \%$ had radiation therapy, and $56.1 \%$ received antihormonal therapy.

The last question of the survey addressed the issue of the general quality of life in terms of how the survivors' quality of life had changed after breast cancer treatment. In total, $42.7 \%$ of the women did not experience a change in their quality of life. Out of the 281 patients with a reported decrease in this global aspect, 238 women (80\%) expressed a change in the field of body image and body awareness and pain. A decrease in their quality of life due to financial problems was pointed out by $19 \%$ of the women, and 31 women (11\%) mentioned problems in their familial surrounding. Multiple answers were possible.

Looking for associations between the quality of life and different factors, the following analysis reflects the sig- nificant correlation $(p<0.05)$. In the group of women with a decreased quality of life due to financial problems, the group of self-employed patients showed this to a higher degree than the women who were employed. Women who did not return to work after the disease experienced a more pronounced decrease in their quality of life than employed women in all analyzed aspects.

\section{Discussion}

We addressed this study in order to evaluate the impact of breast cancer on work-related life and financial factors as well as changes in the general quality of life. To our knowledge, this study is the first to address this question in Germany.

The majority of women diagnosed with breast cancer belong to the working age group. The time it took to return to work after the diagnosis and the treatment of breast cancer are essential points considering the impact of the diagnosis. Returning to work has a great importance for patients and society. For cancer survivors, going back to work underlines a return to normal activities, social recovery, and financial security. In our study, $81.3 \%$ of the patients overall returned to work, $33.7 \%$ of the patients returned to work before 6 months, and in $47.6 \%$ of the cases after 6 months. According to the available literature, these data vary considerably between different countries with different healthcare systems and social insurance systems for regimens [7, 12-14]. For example, breast cancer survivors in the United States show the highest return to work rate (93\%) within 12 months of the diagnosis in comparison to European countries such as the Netherlands with the lowest in the world (43\%). The average reported return-to-work rate is $64 \%$, with a wide range between 24 and $94 \%$. Germany, as other Nordic European countries, provides a comprehensive social system for patients with cancer diagnosis as well as chronic illnesses. The rate of $81.3 \%$ of patients returning to work in Germany seems quite high, and factors as the legal right for cancer patients to participate in a 3-week inpatient cancer rehabilitation program may facilitate returning to work at the earliest possible time. Cancer survivorship and work models published by Feuerstein et al. [15] and Mehnert [16] illustrate the range of individual and interpersonal factors in the short- and late-term effects of cancer treatments as well as the work environment and financial policies that may affect employment and returning to work. National health insurance systems and early or longer disability pensions were found to delay or reduce the return to work, as seen in the Dutch example [14]. Nevertheless, as shown by Maunsell et al. [8], the return to work or giving work up all together is often a personal decision and the women's own choice. 
In our study, $18.7 \%$ of the women never returned to work again. Without knowing the exact factors that mainly influenced the decision, it was one of the strongest predictors for a decrease in the quality of life in all analyzed aspects in our study.

Looking at the literature coming from Japan, unemployment in young patients after a breast cancer diagnosis seems to be an important factor with $29 \%$ losing their jobs and $47.6 \%$ reporting a decrease in their personal income after the diagnosis $[17,18]$. In our cohort of breast cancer patients, $6.5 \%$ lost their job. Data coming from the Nordic countries showed that $5 \%$ of the women were unemployed after the diagnosis of cancer [14].

With regard to financial factors, wage losses constitute an important negative consequence of breast cancer, as discussed in subject literature by many authors. For example, Lauzier et al. [19] reported an average wage loss of $27 \%$ of the usual annual wages, which was most prominent in a lower level of education, receipt of chemotherapy, self-employment, and shorter tenure in the job in a large Canadian study. Our study population experienced a decrease in personal income up to $15 \%$ in $17.6 \%$ of the patients, and $82.4 \%$ reported no change in their wages. Correspondingly, around $18 \%$ of the patients reported a reduction of up to $4 \mathrm{~h}$ per week in working hours, which probably contributes to the reduction of wages, which is in line with the data of Chirikos et al. [20]. In addition to the fact that $17.6 \%$ of the patients reported a decrease in their personal income, more than $30 \%$ felt a reduction of financial opportunities. Previous studies that have included wage losses and out-of-pocket costs indicate that wage losses represent a substantial proportion of the patient's total cost, but out-of-pocket costs probably contribute to the reduction of financial opportunities, as shown by different authors [21, 22]. Zheng at al. [22] described around 5,000 USD of annual excess medical expenditures for breast cancer patients in his panel survey.

With respect to the global quality of life, which was addressed by one item without a detailed questionnaire because of the time-consuming survey for the women, financial problems were named by at least $20 \%$ of the patients to be the major cause for a reduced quality of life. So, therefore, providing a sufficient social system to cover wage losses and improving the survivor's condition and timeline to return to work will affect the overall quality of life in breast cancer survivors, which in fact differs significantly in European countries as well as all over the world.

Long-term, disease-free breast cancer survivors reported a high level of functioning and quality of life many years after primary therapy $[23,24]$. The group of women who reported declined functioning on several dimensions of quality of life was the group who received chemotherapy $[23,24]$. Our data confirmed the influence of che- motherapy on the quality of life as well as on the time to return to work.

With respect to the current study, the results have limitations, which we have to address. First, the beginning of the study period was more than 10 years ago. This may in fact alter the results with respect to medical treatment, health insurance system, and the global or national economic situation. In terms of medical treatment, the rate of given chemotherapy was higher than today, but with respect to the social and employment environment, no significant changes are visible. Second, the survey reflects the respondents' view of cancer's financial and economic impact. This fact is a more subjective measure than assessing the proportion of annual salary loss. Third, our study did not compare the experiences of patients in our sample to non-cancer control patients.

Despite these limitations, the study data provide important information in order to evaluate the impact of breast cancer on work-related life and financial factors, as well as changes in the general quality of life of treated breast cancer patients in Germany.

Better knowledge on work-related problems as well as analyzing the structures that facilitate or interfere with the return to work will help develop necessary interventions for breast cancer survivors.

\section{Conclusion}

Long-term, disease-free breast cancer survivors reported a significant change in their work-related life in terms of the time it took to return to work, unemployment, and the rate of retirement. With respect to financial opportunities, they described a significant decrease with a reported impact on the quality of their life.

\section{Acknowledgments}

The authors would like to thank the participating patients.

\section{Statement of Ethics}

The study was approved by the Committee of Bioethics at Charité, University Medicine Berlin, Germany, and was performed in accordance with the ethical standards laid down in the 1964 Declaration of Helsinki. The participants of the survey gave their informed consent by taking part in the voluntary survey.

\section{Disclosure Statement}

The authors declare that they have no conflicts of interest to disclose. 


\section{Funding Sources}

There was no funding for this study.

\section{Author Contributions}

C.R.-E.: study design, data analysis, manuscript drafting, and final version approval; J.M.P.: data collection, data analysis and final version approval; all authors read and approved the final manuscript.

\section{References}

1 Ferlay J, Soerjomataram I, Ervik M, Dikshit R, Eser S, Mathers C, et al. Globocan 2012 v1.0, Cancer Incidence and Mortality Worldwide: IARC Cancer Case No.11. Lyon, France: International Agency for Research on Cancer; 2013.

2 Stewart BW, Wild CP, editors. World cancer report 2014. Lyon: International Agency for Research on Cancer; 2014.

3 Robert Koch Institut. Bericht zum Krebsgeschehen in Deutschland. Berlin; 2016.

4 Chen L, Linden HM, Anderson BO, Li CI. Trends in 5-year survival rates among breast cancer patients by hormone receptor status and stage. Breast Cancer Res Treat. 2014 Oct; 147(3):609-16.

5 Ghoncheh M, Pournamdar Z, Salehiniya H. Incidence and Mortality and Epidemiology of Breast Cancer in the World. Asian Pac J Cancer Prev. 2016;17(sup3 S3):43-6.

6 Cao SS, Lu CT. Recent perspectives of breast cancer prognosis and predictive factors. Oncol Lett. 2016 Nov;12(5):3674-8.

7 Islam T, Dahlui M, Majid HA, Nahar AM, Mohd Taib NA, Su TT. Factors associated with return to work of breast survivors: a systematic review. BMC Public Health. 2014;14 Suppl 3:S8.

8 Maunsell E, Drolet M, Brisson J, Brisson C, Mâsse B, Deschênes L. Work situation after breast cancer: results from a populationbased study. J Natl Cancer Inst. 2004 Dec; 96(24):1813-22.
9 Yamauchi H, Nakagawa C, Fukuda T. Social impacts of the work loss in cancer survivors. Breast Cancer. 2017 Sep;24(5):694-701.

10 Sun Y, Shigaki CL, Armer JM. Return to work among breast cancer survivors: A literature review. Support Care Cancer. 2017 Mar; 25(3):709-18.

11 Arfi A, Baffert S, Soilly AL, Huchon C, Reyal F, Asselain B, et al. Determinants of return at work of breast cancer patients: results from the OPTISOINS01 French prospective study. BMJ Open. 2018 May;8(5):e020276.

12 Wolvers MD, Leensen MC, Groeneveld IF, Frings-Dresen MH, De Boer AG. Predictors for earlier return to work of cancer patients. J Cancer Surviv. 2018 Apr;12(2):169-77.

13 Mehnert A, de Boer A, Feuerstein M. Employment challenges for cancer survivors. Cancer. 2013 Jun;119 Suppl 11:2151-9.

14 Lindbohm ML, Kuosma E, Taskila T, Hietanen P, Carlsen K, Gudbergsson S, et al. Cancer as the cause of changes in work situation (a NOCWO study). Psychooncology. 2011 Aug;20(8):805-12.

15 Feuerstein M, Todd BL, Moskowitz MC, Bruns GL, Stoler MR, Nassif T, et al. Work in cancer survivors: a model for practice and research. J Cancer Surviv. 2010 Dec;4(4):41537.

16 Mehnert A. Employment and work-related issues in cancer survivors. Crit Rev Oncol Hematol. 2011 Feb;77(2):109-30.

17 Saito N, Takahashi M, Sairenchi T, Muto T. The impact of breast cancer on employment among Japanese women. J Occup Health. 2014;56(1):49-55.
18 Takahashi M. Psychosocial distress among young breast cancer survivors: implications for healthcare providers. Breast Cancer. 2014 Nov;21(6):664-9.

19 Lauzier S, Maunsell E, Drolet M, Coyle D, Hébert-Croteau N, Brisson J, et al. Wage losses in the year after breast cancer: extent and determinants among Canadian women. J Natl Cancer Inst. 2008 Mar;100(5):321-32.

20 Chirikos TN, Russell-Jacobs A, Cantor AB. Indirect economic effects of long-term breast cancer survival. Cancer Pract. 2002 Sep-Oct; 10(5):248-55.

21 Lauzier S, Lévesque P, Mondor M, Drolet M, Coyle D, Brisson J, et al. Out-of-pocket costs in the year after early breast cancer among $\mathrm{Ca}$ nadian women and spouses. J Natl Cancer Inst. 2013 Feb;105(4):280-92.

22 Zheng Z, Yabroff KR, Guy GP Jr, Han X, Li C, Banegas MP, et al. Annual medical expenditure and productivity loss among colorectal, female breast, and prostate cancer survivors in the United States. J Natl Cancer Inst. 2015 Dec;108(5):djv382.

23 Ganz PA, Desmond KA, Leedham B, Rowland JH, Meyerowitz BE, Belin TR. Quality of life in long-term, disease-free survivors of breast cancer: a follow-up study. J Natl Cancer Inst. 2002 Jan;94(1):39-49.

24 Mols F, Vingerhoets AJ, Coebergh JW, van de Poll-Franse LV. Quality of life among longterm breast cancer survivors: a systematic review. Eur J Cancer. 2005 Nov;41(17):2613-9. 